\title{
Reactions of dipivaloylketene and its dimer with C-nucleophiles
}

\author{
Gert Kollenz $^{* a}$, Turkaram S. Dalvi ${ }^{\mathrm{a}}$, Oliver Kappe ${ }^{\mathrm{a}}$ and Curt Wentrup ${ }^{\mathrm{b}}$ \\ ${ }^{a}$ Institut fur Chemie, Karl-Franzens-Universitat Graz, A -8010 Graz, Austria \\ E-mail: kollenz@kfunigraz.ac.at \\ ${ }^{b}$ Department of Chemistry, The University of Queensland, \\ Brisbane, Qid. 4072, Australia
}

(received 02 Dec 99; accepted 13 Feb 00; published on the web 21 Feb 00)

DOI: http://dx.doi.org/10.3998/ark.5550190.0001.111

\begin{abstract}
Dipivaloylketene $\mathbf{1}$ as well as its dimer 2 add 1,3-diketones as C-nucleophiles in strongly basic medium affording the open-chain tetracarbonyl compounds $\mathbf{3}$. The dibenzoylmethane adduct $\mathbf{3 a}$ undergoes acid induced cyclocondensation, and under concomitant loss of one pivaloyl group the 4-pyrone derivative 4 is formed. Employing diethyl malonate as C-nucleophile provides the 2pyrone 5.
\end{abstract}

Keywords: Dipivaloylketene, tetracarbonyl compounds, cyclocondensation

Dipivaloylketene $\mathbf{1}$ is obtained from preparative flash vacuum pyrolysis (FVP) of the corresponding furan-2,3-dione in excellent yield. ${ }^{1}$ It slowly dimerizes to afford the $4 \mathrm{H}-[1,3]-$ dioxin-4-one derivative 2 possessing also an $\alpha$-oxoketene moiety ${ }^{1,2}$. Both compounds are remarkably stable and have been found to smoothly add $\mathrm{N}$ - as well as O-nucleophiles under very mild reaction conditions ${ }^{3}$. The reaction of $\mathbf{1}$ with nucleophiles provides the corresponding dipivaloyl acetic acid derivatives in excellent yields. On the other hand, the products obtained from the reaction of dimer 2 with nucleophiles are 2,9-dioxabicyclo[3.3.1]nona-3,7-dienes featuring the rather rare molecular skeleton of a concave, bridged bisdioxine exhibiting axial chirality ${ }^{3}$. Furthermore, these compounds can be easily transferred by acid hydrolysis into functionalized $\quad 2,4,6, \quad 8$-tetraoxatricyclo[3.3.1.1 $\left.{ }^{3,7}\right]$ decanes $\quad(2,4,6, \quad 8$-tetraoxaadamantanes $)$ (Scheme 1). ${ }^{4}$ 


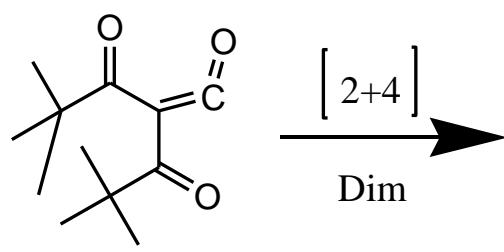

1
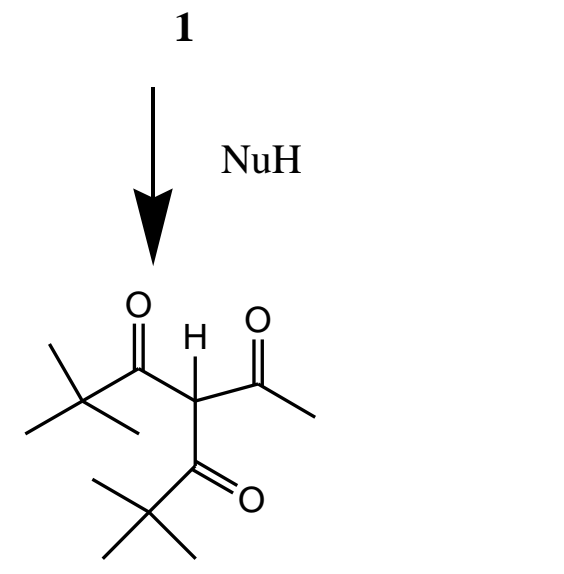<smiles></smiles>

2

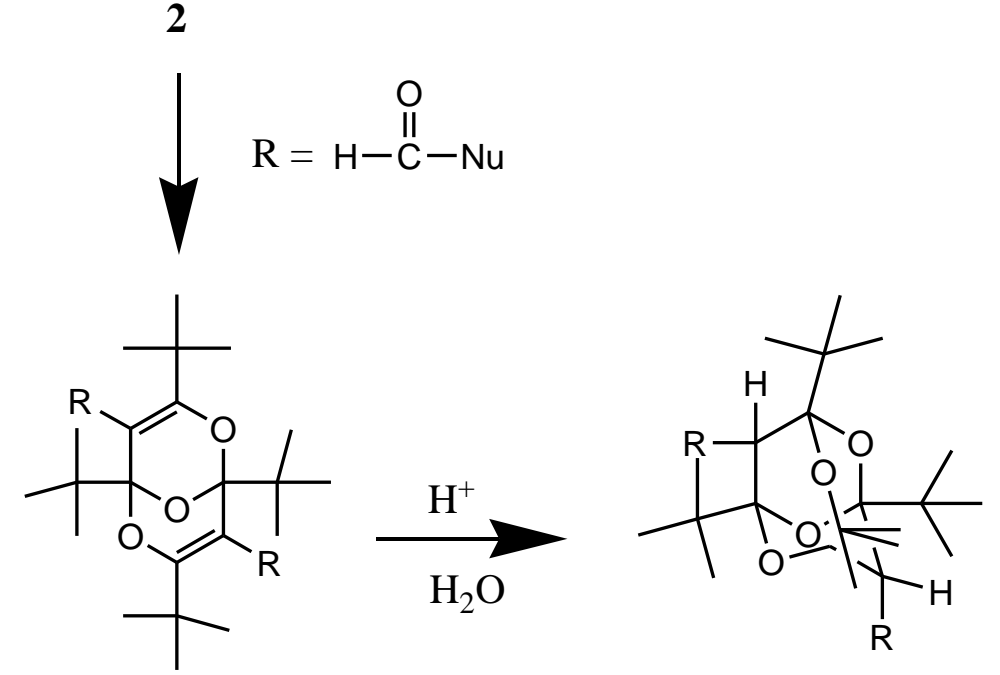

\section{Scheme 1}

These findings prompted the investigation of the reactivity of these stable $\alpha$-oxoketenes 1 and 2 towards carbon-nucleophiles.

\section{Results and Discussion}

1,3-Diketones (dibenzoylmethane, dimesitoylmethane, dipivaloylmethane, and dimedone) were first treated with sodium hydride $(50 \% \text { suspension in mineral oil })^{5}$ in acetonitrile to generate the corresponding carbanions. Subsequently, oxoketenes $\mathbf{1}$ or $\mathbf{2}$ were added at room temperature, and after $24 \mathrm{~h}$ adducts 3 were isolated. Although several tautomeric forms can be envisaged for these products, the tetracarbonyl structure $\mathbf{3}$ (as depicted in Scheme 2) is in agreement with the spectroscopic data: the dipivaloylmethyl moiety of compounds $\mathbf{3}$ is evident from the characteristic $C-H$ signals in the range of $\delta 6.2-6.8$; these shift values indicate a rather strong deshielding due to the anisotropic effects of vicinal carbonyl groups. Products $\mathbf{3}$ do not show any tendency to form other enol tautomers (as evidenced from the NMR spectra and within the accuracy of this method). Earlier measurements of dipivaloylacetic acid derivatives in the solid state and in solution of different solvents demonstrated a similar behaviour. 3b, 6 Further support comes from theoretical investigations on keto-enol tautomerism of $\beta$-tricarbonyl compounds. ${ }^{6}$ The observation of a single $C-H$ signal in structures 3 is in agreement with the corresponding ${ }^{13} \mathrm{C}$ 
NMR signal of $\mathbf{3 a}\left(\delta 64.2, \mathrm{~d},{ }^{1} J=125 \mathrm{~Hz}\right)$ and leads to the conclusion that only the monoenol forms 3 are resent.

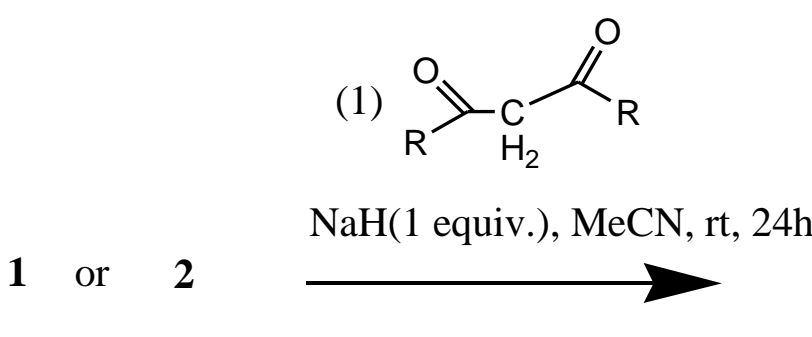

(2) aq $\mathrm{HCl}$

3a: $\mathrm{R}=\mathrm{Ph}$

3b: $\mathrm{R}=$

3c: $\mathrm{R}=\mathrm{t}-\mathrm{Bu}$

3d: $\mathrm{R}=\mathrm{X}_{\mathrm{Me}}^{\mathrm{Me}}$

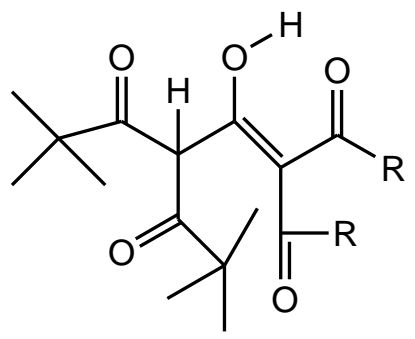

3

3a

PPA, $90^{\circ} \mathrm{C}, 4 \mathrm{~h}$

$-\mathrm{H}_{2} \mathrm{O}$

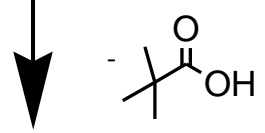<smiles>CC(C)(C)c1cc(=O)c(C(=O)c2ccccc2)c(-c2ccccc2)o1</smiles>

4

\section{Scheme 2}

The experimental findings that compounds 3 were also obtained from the reaction of the xoketene 2 confirms earlier results ${ }^{3 b}$ that strong bases (e.g. triethylamine ${ }^{3 b}$ ) catalyze the dissociation of the dioxinone ring into two monomers of ketene 1. Attempts to cyclize the openchain compounds $\mathbf{3}$ were successful only in case of $\mathbf{3 a}$; treatment with polyphosphoric acid at $90^{\circ}$ $\mathrm{C}$ afforded the 4-pyrone derivative 4. Under the reaction conditions applied, one pivaloyl group was lost. Examples for similar deacylation reactions of polyacyl compounds in acidic media are well known. ${ }^{7}$ Allring carbon atoms of $\mathbf{4}$ are readily assigned from their ${ }^{13} \mathrm{C}-\mathrm{NMR}$ signals.

Surprisingly, when oxoketenes 1 or $\mathbf{2}$ were treated with ethyl malonate and $\mathrm{NaN}$, no open-chain adduct could be isolated, the 4-hydroxy-2-pyrone derivative 5 was the only reaction product obtained. Its structure was elucidated mainly by evaluation of its ${ }^{13} \mathrm{C}-\mathrm{NMR}$ data. A plausible reaction mechanism for the formation of $\mathbf{5}$ is given in Scheme 3. 


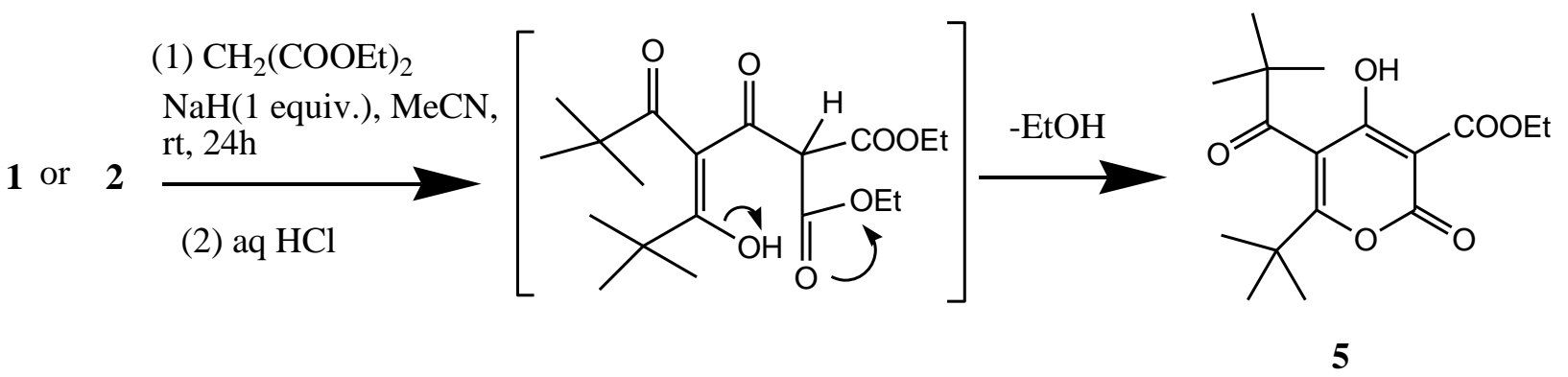

\section{Scheme 3}

In summary, the reaction of the stable $\alpha$-oxoketenes $\mathbf{1}$ and $\mathbf{2}$ with $\mathrm{C}$-nucleophiles gives rise to $\mathrm{C}$ $\mathrm{C}$ bond formation leading to tetracarbonyl compounds $\mathbf{3}$ and / or pyrone derivatives $\mathbf{4}$ and $\mathbf{5}$ in moderate to good yields. In order to generate the required carbanions, the reactions have to be performed in the presence of a strong base, which in case of the dimer $\mathbf{2}$ also initiates a cycloreversion of the dioxinone ring ${ }^{3 b}$. Formation of bridged bisdioxines (see Scheme 1) was not observed in the course of this work.

\section{Experimental Section}

General Procedures. IR spectra ( $\mathrm{KBr}$ disks) were recorded on a Perkin Elmer 298 Spectrometer, ${ }^{1} \mathrm{H}$ and ${ }^{13} \mathrm{CNMR}$ (gated decoupling mode) spectra were recorded on a Varian $\mathrm{XL}$ $200 \mathrm{Gemini}$ Spectrometer at $200 \mathrm{MHz}$ and $50 \mathrm{MHz}$, respectively. Elemental Analyses were obtained from a CNN Elemental Analyzer Carlo Erba 1106. $\alpha$-Oxoketenes 1 and 2 were prepared according to the literature. ${ }^{1,2}$

\section{General procedure for the reactions of $\alpha$-oxoketenes 1 and 2 with $\mathbf{C}$-nucleophiles}

The monosodium salts of 1,3-diketone and diethyl malonate were prepared ${ }^{5}$ in situ by adding the active methylene compound $(0.27-0.71 \mathrm{mmol}$ ) to a stirred suspension of NaN (equimolar in $2 \mathrm{~mL}$ of acetonitrile) at $\mathrm{rt}$ during $10-15 \mathrm{~mm}$. To this suspension the $\alpha$-oxoketene 1 or 2 equimolar amount) was added at once, and stirring was continued at $\mathrm{rt}$. After $24 \mathrm{~h}$ the mixture was diluted with $\mathrm{H}_{2} \mathrm{O}(5 \mathrm{~mL})$, neutralized with aqueous $\mathrm{HCl}$, and extracted with dichloromethane (4 times $\mathrm{S} \mathrm{mL}$ ). The combined organic layers were dried over $\mathrm{Na}_{2} \mathrm{SO}_{4}$ for $4 \mathrm{~h}$. After filtration and evaporation of the solvent the colourless crude residue was recrystallized from petroleum ether $\left(60-80{ }^{\circ} \mathrm{C}\right)$ or petroleum ether / ethyl acetate.

\section{2-Benzoyl-3-hydroxy-6,6-dimethyl-4-(2,2-dimethylpropanoyl)-l-phenylhept-2-ene-l,5d-ione} (3a). a. From dibenzoylmethane $(160 \mathrm{mg}, 0.71 \mathrm{mmol})$ and $1(150 \mathrm{mg}, 0.71 \mathrm{mmol})$ pure 3a (120 mg, 79\%) was isolated; $\mathrm{mp}$ 136-137 ${ }^{0} \mathrm{C}$ (petroleum ether/ethyl acetate 3:1). b. Dibenzoylmethane (112 mg, $0.5 \mathrm{mmol})$ and $2(210 \mathrm{mg}, 0.5 \mathrm{mmol})$ gave $3 \mathbf{a}(140 \mathrm{mg}, 65 \%)$. JR 
(KiBr): 3000-2800, 1720, 1700, 1650, 1600, $1550 \mathrm{~cm}^{-1} .{ }^{1} \mathrm{HNMR}\left(\mathrm{CDCI}_{3}\right): \delta 1.10,(\mathrm{~s}, 18 \mathrm{H}), 6.35$ (s, iN), 7.03-7.65 (in, $1 \mathrm{iN}) .{ }^{13} \mathrm{CNMR}\left(\mathrm{CDCl}_{3}\right): \delta 207.47$ (in, $\mathrm{C}=\mathrm{O}$ pivaloyl), $196.6\left(\mathrm{t},{ }^{3} \mathrm{~J}=3.5 \mathrm{~Hz}\right.$, C=O benzoyl), 193.5 (dd, J 4.5 Hz, J $2.5 \mathrm{~Hz}, \mathrm{C}-3$ ), 185.45 (dt, ${ }^{3} J 3.5 \mathrm{~Hz}, \mathrm{C} 0$ benzoyl), 138.6, 134.7, 133.0, 132.16, 129.46, 128.8, 128.3, 128.2 (C arom.), 113.8 (d, $\left.{ }^{3} J=4.5 \mathrm{~Hz}, \mathrm{C}-2\right), 64.3$ (d, $\left.{ }^{1} J=\mathrm{Hz}, \mathrm{C}-4\right)$, 45.4 (in, $\left.\underline{\mathrm{C}}\left(\mathrm{CH}_{3}\right)_{3}\right)$, 27.1, (s, $\left.\mathrm{C}\left(\underline{\mathrm{CH}}_{3}\right)_{3}\right)$. Anal. Calcd. for $\mathrm{C}_{27} \mathrm{H}_{30} \mathrm{O}_{5}$ : C, 74.63; H, 6.96. Found: C, 74.68; N, 6.95 .

3-Hydroxy-6,6-dimethyl-4-(2,2-dimethylpropanoyl)-2-(2,4,6-trimethylbenzoyl)-1-(2,4,6trimethylphenyl)hept-2-ene-1,5-dione (3b). a. Dimesitoylmethane (160 mg, 0.S2 mmol) and 1 (110 mg, 0.S2 mmol) afforded 3b (200 mg, 77\%); mp 138-139 ${ }^{0} \mathrm{C}$ (petroleum ether / ethyl acetate 5:1). b. Dimesitoylmethane (150 mg, $0.49 \mathrm{mmol})$ and 2 (200 $\mathrm{mg}, 0.49 \mathrm{mmol})$ gave 3b (140 mg, 57\%). IR(KBr): 3060-2800, 1720, 170S, 1640, 1610, $1480 \mathrm{~cm}^{-1} .{ }^{1} \mathrm{HNMR}\left(\mathrm{CDCl}_{3}\right): \delta$ $1.27(\mathrm{~s}, 18 \mathrm{~N}), 1.98(\mathrm{~s}, 6 \mathrm{~N}), 2.02(\mathrm{~s}, 6 \mathrm{~N}), 2.14(\mathrm{~s}, 3 \mathrm{~N}), 2.16(\mathrm{~s}, 3 \mathrm{~N}), 6.46(\mathrm{~s}, 2 \mathrm{H}), 6 . \mathrm{SO}(\mathrm{s}, 2 \mathrm{~N})$, 6.77 (s, iN). Anal. Calcd. for $\mathrm{C}_{33} \mathrm{~N}_{42} \mathrm{O}_{5}$ : C, 76.41; N, 8.16. Found: C, 76.19; N, 8.10.

5-Hydroxy-4,6-di(2,2-dimethylpropanoyl)-2,2,8,8-tetramethylnon-4-ene-3,7-dione (3c). a. From dipivaloylmethane (100 mg, $0.54 \mathrm{mmol})$ and 1 (120 mg, $0.54 \mathrm{mmol}) \mathbf{3 c}(80 \mathrm{mg}, 37 \%)$ was isolated; $\mathrm{mp} 117-118{ }^{\circ} \mathrm{C}$ (petroleum ether). b. From dipivaloylmethane $(50 \mathrm{mg}, 0.27 \mathrm{mmol})$ and 2 (120 mg, $0.27 \mathrm{mmol}) 3 \mathbf{c}(40 \mathrm{mg}, 37 \%)$ was obtained. JR (KBr): 3040-2800, 1760, 1720, 169S, 1630, $1475 \mathrm{~cm}^{1} .{ }^{1} \mathrm{H}-\mathrm{NMR}\left(\mathrm{CDCl}_{3}\right): \delta 1.13(\mathrm{~s}, 9 \mathrm{H}), 1.18(\mathrm{~s}, 18 \mathrm{H}), 1.30(\mathrm{~s}, 9 \mathrm{~N}), 5.55($ broad s, $1 \mathrm{~N}$, $\mathrm{ON}), 6.20$ (s, iN). Anal. Calcd. for $\mathrm{C}_{23} \mathrm{~N}_{38} 0_{5}$ : C, 70.02; N, 9.71. Found: C, 69.96; N, 9.45.

4-[(4,4-Dimethyl-2,6-dioxocyclohexyliden)(hydroxy)methyl]-2,2,6,6-tetramethylheptane 3,5-dione (3d). a. Dimedone (70 mg, $0.5 \mathrm{mmol})$ and $1(105 \mathrm{mg}, 0.5 \mathrm{mmol})$ gave $3 \mathrm{~d}(120 \mathrm{mg}$, $6 \mathrm{~S} \%$ ); mp 71-72 ${ }^{0} \mathrm{C}$ (petroleum ether). b. Dimedone (70 mg, $0.5 \mathrm{mmol}$ ) and 2 (210 mg, 0.5 mmol) yielded 3d (130 mg, 70\%). JR (KBr): 3040-2800, 1720, 1700, 1665, 1570, 1490, 1475 $\mathrm{cm}^{1} .{ }^{1} \mathrm{H}-\mathrm{NMR}\left(\mathrm{CDCl}_{3}\right): \delta 1.06(\mathrm{~s}, 6 \mathrm{H}), 1.16(\mathrm{~s}, 18 \mathrm{H}), 2.32(\mathrm{~s}, 2 \mathrm{~N}), 2 . \mathrm{S} 7(\mathrm{~s}, 2 \mathrm{~N}), 6.86(\mathrm{~s}, 1 \mathrm{~N})$. Anal. Calcd. for $\mathrm{C}_{20} \mathrm{H}_{30} 0_{5}$ : C, 68.55; H, 8.63. Found: C, 68.02; H, 8.62.

3-Benzoyl-6-t-butyl-2-phenyl-4H-pyran-4-one (4). A mixture of 3a (170 mg, $0.39 \mathrm{mmol}$ ) and polyphosphoric acid ( $2 \mathrm{~g}$, excess) was heated at $85-90{ }^{0} \mathrm{C}$ with occasional stirring for $4 \mathrm{~h}$. After cooling to $20{ }^{0} \mathrm{C} \mathrm{N}_{2} \mathrm{O}(\mathrm{S} \mathrm{mL})$ was added, and the reaction mixture was stirred for $2 \mathrm{~mm}$. Further dilution with $\mathrm{H}_{2} \mathrm{O}(20 \mathrm{~mL})$ was followed by extraction with dichloromethane $(3 \mathrm{x} 10 \mathrm{~mL})$. The combined organic layers were dried over $\mathrm{Na}_{2} \mathrm{SO}_{4}$. After the solvent was removed on a rotary evaporator the colourless solid obtained crystallized from petroleum ether / ethyl acetate (4:1) to give 4 (90 mg, 65\%); mp 186-187 ${ }^{\circ} \mathrm{C}$. IR(KBr): 3000-2800, 1670, 164S, 1610, 1600, 1S80, 1490, 14S0 cm ${ }^{1} .{ }^{1} \mathrm{H}-\mathrm{NMR}\left(\mathrm{CDCl}_{3}\right): \delta 1.40(\mathrm{~s}, 9 \mathrm{H}), 6.4(\mathrm{~s}, 1 \mathrm{~N}), 7.28,8.0$ (in, $\left.1 \mathrm{ON}\right) .{ }^{13} \mathrm{C}-\mathrm{NMR}\left(\mathrm{CDCl}_{3}\right)$ : $\delta 193.8\left(\mathrm{t},{ }^{3} \mathrm{~J}=4.5 \mathrm{~Hz}\right.$, benzoyl C=O), 178.6 (s, C-4), 175.9 (in, C-6), 161.8 (t, ${ }^{3} J=5.5 \mathrm{~Hz}, \mathrm{C}-2$ ), 137.0, 134.5, 131.5, 130.0, 129. 2, 128.0 (arom. C), 125.5 (d, $\left.{ }^{3} J=3.5 \mathrm{~Hz}, \mathrm{C}-3\right), 110.5$ (d, ${ }^{1} J=$ $162.5 \mathrm{Nz}, \mathrm{C}-5), 36.5\left(\mathrm{~m}, \underline{\mathrm{C}}\left(\mathrm{CH}_{3}\right)_{3}\right), 28.0$ (q, $\left.{ }^{1} \mathrm{~J}=125.0 \mathrm{~Hz}, \mathrm{C}\left(\underline{\mathrm{C}} \mathrm{H}_{3}\right)_{3}\right)$. Anal. Cald. for $\mathrm{C}_{22} \mathrm{~N}_{20} \mathrm{O}_{3}$ : C; 79.50; N, 6.06. Found: C, 79.74; H, 6.07.

Ethyl 6-(t-butyl)-4-hydroxy-5-(2,2-dimethylpropanoyl)-2-oxo-2H-pyran-3-carboxylate (5) a. Diethyl inalonate $(120 \mathrm{mg}, 0.73 \mathrm{mmol})$ and 1 (150 mg, $0.71 \mathrm{inmol})$ gave 5 (130 ing, 54\%); mp $55-56{ }^{\circ} \mathrm{C}$ (petroleum ether). b. Diethyl malonate $(80 \mathrm{mg}, 0.5 \mathrm{inmol})$ and 2 (200 $\left.\mathrm{mg}, 0.475 \mathrm{minol}\right)$ 
afforded 5 (80 ing, 9\%). IR(KBr): 3040-2800, 1750, 1700, 1640, 1610, $1545 \mathrm{~cm}^{1} .{ }^{1} \mathrm{H}-\mathrm{NMR}$ $\left(\mathrm{CDCl}_{3}\right): \delta 1.28(\mathrm{~s}, 18 \mathrm{~N}), 1.42(\mathrm{t}, J=7.5 \mathrm{~Hz}, 3 \mathrm{~N}), 4.47(\mathrm{q}, J=7.5 \mathrm{~Hz}, 2 \mathrm{~N}) \cdot{ }^{13} \mathrm{C}-\mathrm{NMR}\left(\mathrm{CDCl}_{3}\right): \delta$ 209.6 (m, C=O pivaloyl),3 177.2 (d, $\left.{ }^{2} J=4.5 \mathrm{~Hz}, \mathrm{C}-4\right), 172.9$ (m, C-6), 171.7 (t, $J 3 \mathrm{Nz}, \mathrm{C} 0$ ethoxycarbonyl), 157.1 (s, C-2), 114.2 (d, $\left.{ }^{3} J=7 \mathrm{~Hz}, \mathrm{C}-5\right), 91.0\left(\mathrm{~d},{ }^{3} J 4.5 \mathrm{~Hz}, \mathrm{C}-3\right), 62.8\left(\mathrm{t},{ }^{1} J=\right.$ $\left.150 \mathrm{~Hz}, \mathrm{OCN}_{2}\right), 45.5,38.7\left(\mathrm{~m}, \underline{\mathrm{C}}\left(\mathrm{CH}_{3}\right)_{3}\right), 29.0,28.6\left(\mathrm{q},{ }^{1} J=125 \mathrm{~Hz}, \mathrm{C}\left(\underline{\mathrm{CN}}_{3}\right)_{3}\right), 14.2\left(\mathrm{q},{ }^{1} J=125\right.$ $\mathrm{Hz}, \mathrm{CH}_{3}$ ). Anal. Cald. for $\mathrm{C}_{17} \mathrm{H}_{24} \mathrm{O}_{6}$ : C, 62.95; N, 7.46. Found: C, 63.00; N, 7.41.

\section{References}

1. Kappe, C.O.; Evans, R.A.; Kennard, C.H.L; Wentrup, C. J. Am. Chem. Soc. 1991, 113, 4234.

2. Kappe, C.O.; Farber, G.; Wentrup, C.; Kollenz, G. I Org. Chem. 1992, 57, 7078.

3. (a) Kappe, C.O.; Farber, G.; Wentrup, C.; Kollenz, G. Tetrahedron Lett. 1992, 33, 4543. (b) Kappe, C.O., Kollenz, G.; Fabian, W.M.F.; Wentrup, C.; Farber G. J. Org. Chem. 1993, 58, 3361.

4. (a) Neilmayer, W.; Dalvi, T.S.; Kappe, C.O.; Wentrup, C.; Gruber, K.; Sterk, H.; Kollenz, G. I Chem. Soc., Chem. Comm. 1995, 797. (b) Dalvi, T.S.; Kappe, C.O.; Wentrup, C.; Kollenz, G. Heterocycles 1998, 48, 1841.

5. (a) Z.; Najos, G.; Kollenz, G.; Messmer, A. Chem. Ber. 1994, 127, 1799. (b) Fieser, L.; Fieser, M., Eds.: Reagents for Organic Synthesis, J.Wiley \& Sons: New York, 1967; Vol.1, p 1075.

6. Fabian, W.M.F.; Kollenz, G.; Akcamur, Y.; K6k, T.R.; Teczan, M.; Akkurt, M.; Niller, W. Monatsh. Chem. 1992, 123, 265. (b) Fabian, W.M.F.; Trinkel, M.; Perjessy, A. I Mol. Struct. 1994, $317,1$.

7. (a) Ott,W.; Ziegler, E.; Kollenz, G. Synthesis 1976, 477. (b) Kollenz, G.; Kriwetz, G.; Ott, W.; Ziegler, E. Liebigs Ann. Chem. 1977, 1964.

8. Kohler, E.B.; Barnes C.E. J. Am. Chem. Soc. 1933, 55, 690. 